\title{
A Prospective Comparative Study of Twist Drill Craniostomy Versus Burr Hole Craniostomy in Patients with Chronic Subdural Hematoma
}

\author{
Ke WANG*, Dongjiang $\mathrm{CHEN}^{*}$, Xiangyuan CAO, Liang GAO \\ Tongji University, Shanghai Tenth People's Hospital, Department of Neurosurgery, China \\ *Ke WANG and Dongjiang CHEN contributed equally to this work and should be considered jointly as first authors.
}

\section{ABSTRACT}

AIM: This study aims to compare clinical outcomes in patients with chronic subdural hematoma (CSDH) following twist drill craniostomy (TDC) or burr hole craniostomy (BHC).

MATERIAL and METHODS: A prospective cohort study was conducted in the patients who suffered from symptomatic CSDH and received surgical treatment in our department from Jan 2011 to Dec 2013. Each patient was followed 3 months after the surgery.

RESULTS: Thirty-eight and 45 patients received TDC and BHC treatment, respectively. There was no significant difference in age, gender, head trauma, diabetes mellitus, hypertension, antiplatelet usage, clinical manifestation, the Glasgow Coma Scale score and preoperative radiographic characteristics between the two groups. Patients in TDC had a significantly shorter operating time, but a longer draining time than those in $B H C(16.9 \pm 6.3$ min vs. $44.4 \pm 7.1 \mathrm{~min}, \mathrm{p}<0.001 ; 3.1 \pm 1.0 \mathrm{~d}$ vs. $2.5 \pm 0.9 \mathrm{~d}$, $\mathrm{p}=0.003$; respectively). A smaller degree of midline shift reversal was observed in patients after TDC than those after BHC $(2.6 \pm 2.5 \mathrm{~mm}$ vs. $3.9 \pm 2.8, \mathrm{p}=0.030)$. Seven patients $(18.4 \%)$ in TDC and 5 patients $(11.1 \%)$ in BHC experienced CSDH recurrence. There was no significant difference in the recurrence rate, in-hospital complications, and neurological outcomes between the two groups.

CONCLUSION: This study indicates that TDC and BHC have similar clinical outcomes in the treatment of patients with CSDH. A shorter operating time, but a smaller midline shift reversal and a longer draining time may be expected in patients after TDC than after BHC.

KEYWORDS: Subdural hematoma, Chronic subdural hematoma, Twist drill craniostomy, Burr hole craniostomy, Recurrence

\section{INTRODUCTION}

$\longrightarrow$ hronic subdural hematoma (CSDH) is a neurological disorder that predominantly affects elderly people and causes serious morbidity and mortality $(1,2)$. Surgical treatment plays an important role in the treatment of patients with symptomatic CSDH. Twist drill craniostomy (TDC), burr hole craniostomy (BHC), and craniotomy are three commonly used procedures in current clinical practice. Among them, TDC and BHC are recognized as first-tier surgical treatment while craniotomy is used as a second-tier treatment (16). Both TDC and BHC can be performed in setting of local anesthesia, and TDC can even be performed at bedside (7). Most neurosurgeons tend to use $\mathrm{BHC}$ as the procedure of choice to treat patients with initial CSDH (3). With a smaller diameter of skin incision and skull opening, TDC is less invasive than $\mathrm{BHC}$, which theoretically favors its use in elderly patients who usually have comorbidities. Previous studies comparing therapeutic effects of TDC and $\mathrm{BHC}$ concluded controversial results $(6-8,17)$. Here, we conducted a prospective cohort 
study to compare clinical outcomes of the two surgical techniques in a selected group of patients with symptomatic $\mathrm{CSDH}$.

\section{MATERIAL and METHODS}

\section{Inclusion and Exclusion Criteria}

A prospective cohort study was undertaken in the patients who suffered from symptomatic CSDH and admitted to the Neurosurgical Department, Shanghai Tenth People's Hospital between January 2011 and December 2013. Inclusion criteria of patients in the study were as follows: (a) patient age $\geq$ 18 years old; (b) initial unilateral CSDH without loculation confirmed by computed tomography (CT); (c) patients who received either TDC or $\mathrm{BHC}$ treatment; (d) patients with written consent from their next of kin. Patients with recurrent or bilateral $\mathrm{CSDH}$ were excluded from this study. Patients with severe systemic diseases, such as chronic heart failure, coagulation disorders, thrombocytopenia, respiratory insufficiency, liver or renal dysfunction, were excluded. Patients dependent on anticoagulants or alcohol were also excluded. The Institutional Review Board approved this study.

\section{TDC and BHC Procedures}

Each patient received head CT scan before surgery. Based on individual preference, the neurosurgeons performed either TDC with drainage or BHC with drainage for each patient in the operating room. All the operations were performed under local anesthesia with $2 \%$ lidocaine, and adjunctive intravenous anesthesia with propofol and fentanyl was added if the patient was uncooperative. A single-shot antibiotic prophylaxis with first generation cephalosporin was intravenously administered $30 \mathrm{~min}$ before skin incision. The single-use $\mathrm{YL}-1$ puncture needle for treatment of encephalic hematomas (Beijing Wantefu Medical Apparatus Corporation, China) was used for the patients who received TDC treatment. With a 3-mm external and $2.5-\mathrm{mm}$ internal diameter, the needle has an available length ranging from $20 \mathrm{~mm}$ to $70 \mathrm{~mm}$ with an increment of $5 \mathrm{~mm}$. The preoperative CT image was used to measure the distance from the skin surface to the outer membrane of the hematoma in order to determine the appropriate length of the needle. Under sterile conditions and local anesthesia, a 4-5 $\mathrm{mm}$ skin incision was made over the maximum width of the hematoma and a craniostomy was made using an electronic drill with the needle attached on it. After the needle penetrated the outer membrane of the hematoma, the neurosurgeon felt resistance loss and removed the stylet inside the needle to allow for outflow of liquefied hematoma. A closed external ventricular drainage system was attached to the trocar port on the needle.

In the patients who underwent $\mathrm{BHC}$, a 3-4 cm skin incision was made over the area of maximal hematoma thickness. A single burr hole was made using a 13-mm Cushing drill. After coagulation with bipolar diathermy, the dura and outer membrane was incised in cruciate fashion. The hematoma cavity was irrigated by warm saline solution at body temperature. After that, an external ventricular draining tube was inserted into the hematoma cavity and tunneled subcutaneously to prevent infection. The same closed external ventricular drainage system was used in these patients.

\section{Postoperative Care}

After surgery, all patients were kept at the supine position, with the chamber of the closed drainage system at $40-50 \mathrm{~cm}$ below the head level. Patients were encouraged to do active lower limb movement in bed and to cough out sputum. The drainage system was removed when draining volume per 8 hours was less than $5 \mathrm{~mL}$. Antibiotic prophylaxis was used during the draining period. Each patient routinely received head CT scan within 24 hours after surgery, on the day of draining system removal, at discharge, and at 3-month follow-up. Additional CT would be obtained if patients had deteriorated neurological conditions during this period. Antiplatelet agent was restarted at the day of discharge for the patients who had used it preoperatively.

Recurrence referred to clinical and radiographic worsening due to enlargement or reappearance of a previously treated $\mathrm{CSDH}$ within 3 months after the initial surgery. The recurrence rate was defined as the rate of reoperation to treat a recurrent $\mathrm{CSDH}$ during the time period. Functional outcome was measured by the Glasgow Outcome Scale (GOS) and modified Rankin Scale (mRS) at 3-month follow-ups. A GOS score $\leq 3$ and a $\mathrm{mRS}$ score $\geq 4$ were regarded as an unfavorable outcome.

\section{Statistical Analysis}

Data collected included patients' demographics, radiographic characteristics, and medical histories. The internal structure of the hematoma was classified into four types as previously described: homogenous, laminar, separate or trabecular $(4,9,11)$. Continuous data were expressed in the mean \pm standard deviation (SD) while categorical data was described as the percentage. All statistical analyses were performed using the SPSS 16.0 for windows. The two-tailed student's $t$ test, the Mann-Whitney $U$ test, the Pearson Chi-square test, or the Fisher's exact test was used to evaluate the differences of variables, which were considered significant at $p<0.05$.

\section{- RESULTS}

A total of 83 patients were enrolled, with a mean age of 66 years. As shown in Table I, sixty-six male and 17 female patients were included. Among them, forty-two patients suffered from left CSDH while 41 patients had right $\mathrm{CSDH}$. Thirty-eight patients received TDC and 45 patients underwent $\mathrm{BHC}$ treatment. There was no significant difference in age, gender, head trauma, diabetes mellitus, hypertension, antiplatelet usage, clinical manifestation, the Glasgow Coma Scale (GCS) score, and preoperative CT characteristics between the two groups of patients.

As shown in Table II, patients who received TDC treatment had a significantly shorter operating time than those who received $\mathrm{BHC}$ plus drainage $(16.9 \pm 6.3 \mathrm{~min}$ vs. $44.4 \pm 7.1 \mathrm{~min}$, $\mathrm{p}<0.001)$. However, the draining time in TDC was longer than that in $\mathrm{BHC}(3.1 \pm 1.0 \mathrm{~d}$ vs. $2.5 \pm 0.9 \mathrm{~d}, \mathrm{p}=0.003)$. Head CT scans showed that both TDC and $\mathrm{BHC}$ significantly reduced the midline shift after surgery $(6.4 \pm 4.1 \mathrm{~mm}$ vs. $3.8 \pm 2.3 \mathrm{~mm}$, $\mathrm{p}=0.003 ; 7.9 \pm 4.5 \mathrm{~mm}$ vs. $4.1 \pm 2.6 \mathrm{~mm}, \mathrm{p}<0.001$ respectively). However, the degree of midline shift reversal was significantly 
smaller in TDC than that in BHC $(2.6 \pm 2.5 \mathrm{~mm}$ vs. $3.9 \pm 2.8$ $\mathrm{mm}, \mathrm{p}=0.030$ ) although there was no significant difference in postoperative midline shift between the two groups. Moreover, $\mathrm{BHC}$ significantly reduced the percentage of patients with the midline shift $\geq 5 \mathrm{~mm}$ from $71.1 \%$ to $40.0 \%$ ( $p=0.003$ ) while TDC reduced the percentage from $63.2 \%$ to $44.7 \%$, showing no significant difference $(p=0.107)$.

Seven patients in TDC and 5 patients in $\mathrm{BHC}$ had their $\mathrm{CSDH}$ reoccurred during the observation period, demonstrating not significant difference $(18.4 \%$ vs. $11.1 \%, p=0.345)$. All the twelve patients received reoperation using the same technique as the initial treatment. There was no significant difference in in-hospital complications between the two groups of patients. Neither the GOS score nor the mRS score at 3-month followup demonstrated significant difference between them. One patient in TDC died of uncontrolled pneumonia during hospitalization.

\section{Illustrative Case}

An 80-year-old male patient suffered from headache for two days and came to the emergency department. The history review showed that he had mild head trauma due to falling two weeks ago. At admission, he was stuporous, aphasic, and incontinent. His right-sided muscle strength decreased to grade 2 using the Medical Research Council Scale. The head CT displayed a large left CSDH with significant midline shift towards the opposite side (Figure 1A). He underwent an emergent surgery of TDC with drainage, and the postoperative CT scan showed remarkable reduction of the hematoma volume and subtotal reversal of the midline shift (Figure 1B). The draining volume was $300 \mathrm{~mL}$ in the first day and $100 \mathrm{~mL}$ in the second day after surgery. The draining system was removed in the third day, and the patient was discharged on the fourth day after surgery. He was alert, continent, and showed total recovery of right-sided muscle strength to grade 5 . The pre-discharge CT showed the total reversal of

Table I: General Characteristics of Enrolled Patients

\begin{tabular}{|c|c|c|c|c|}
\hline & $\begin{array}{c}\text { All patients } \\
(n=83)\end{array}$ & $\begin{array}{c}\text { TDC } \\
(\mathrm{n}=38)\end{array}$ & $\begin{array}{c}\text { BHC } \\
(n=45)\end{array}$ & $p$ value \\
\hline Gender, Male & $66(79.5 \%)$ & $28(73.7 \%)$ & $38(84.4 \%)$ & 0.226 \\
\hline \multicolumn{5}{|l|}{ Medical history } \\
\hline Head trauma & $49(59.0 \%)$ & $23(60.5 \%)$ & $26(57.8 \%)$ & 0.800 \\
\hline Hypertension & $33(39.8 \%)$ & $18(47.4 \%)$ & $15(33.3 \%)$ & 0.193 \\
\hline Antiplatelets & $12(14.5 \%)$ & $6(15.8 \%)$ & $6(13.3 \%)$ & 0.751 \\
\hline \multicolumn{5}{|l|}{ Preoperative GCS score } \\
\hline Mean \pm SD & $14.4 \pm 1.6$ & $14.5 \pm 1.3$ & $14.5 \pm 1.8$ & 0.891 \\
\hline $14-15$ & $75(90.4 \%)$ & $34(89.5 \%)$ & $41(91.1 \%)$ & 1.000 \\
\hline Decreased consciousness & $18(21.7 \%)$ & $9(23.7 \%)$ & $9(20.0 \%)$ & 0.685 \\
\hline Headache & $39(47.0 \%)$ & $18(47.4 \%)$ & $21(46.7 \%)$ & 0.949 \\
\hline Focal neurological deficit & $44(53.0 \%)$ & $23(60.5 \%)$ & $21(46.7 \%)$ & 0.208 \\
\hline \multicolumn{5}{|c|}{ Preoperative radiographic features } \\
\hline Laterality, Left & $42(50.6 \%)$ & $18(47.4 \%)$ & 24 (53.3\%) & 0.588 \\
\hline Homogenous type & $39(47.0 \%)$ & $20(52.6 \%)$ & $19(42.2 \%)$ & 0.344 \\
\hline Laminar type & $26(31.3 \%)$ & $16(42.1 \%)$ & $20(44.4 \%)$ & 0.830 \\
\hline Separate type & $18(21.7 \%)$ & $9(23.7 \%)$ & $9(20.0 \%)$ & 0.685 \\
\hline
\end{tabular}


Table II: Outcome and Complications

\begin{tabular}{|c|c|c|c|}
\hline & $\operatorname{TDC}(\mathrm{n}=38)$ & $\mathrm{BHC}(\mathrm{n}=45)$ & p value \\
\hline \multicolumn{4}{|l|}{ Operating time, (min) } \\
\hline Mean \pm SD & $16.9 \pm 6.3$ & $44.4 \pm 7.1$ & $<0.001$ \\
\hline \multicolumn{4}{|l|}{ Postoperative midline shift, (mm) } \\
\hline$\underline{\text { Mean } \pm \mathrm{SD}}$ & $3.8 \pm 2.3$ & $4.1 \pm 2.6$ & 0.666 \\
\hline$<5$ & $21(55.3 \%)$ & $27(60.0 \%)$ & 0.189 \\
\hline $5-10$ & $17(44.7 \%)$ & $17(37.8 \%)$ & 0.521 \\
\hline$>10$ & 0 & $1(2.2 \%)$ & \\
\hline \multicolumn{4}{|l|}{ Change of the midline shift, $(\mathrm{mm})$} \\
\hline Mean \pm SD & $2.6 \pm 2.5$ & $3.9 \pm 2.8$ & 0.030 \\
\hline \multicolumn{4}{|l|}{ Postoperative GCS score } \\
\hline Mean \pm SD & $14.3 \pm 2.2$ & $14.9 \pm 0.3$ & 0.106 \\
\hline $14-15$ & $35(92.1 \%)$ & $44(97.8 \%)$ & 0.328 \\
\hline $9-13$ & $2(5.3 \%)$ & $1(2.2 \%)$ & 0.591 \\
\hline$\leq 8$ & $1(2.6 \%)$ & 0 & \\
\hline \multicolumn{4}{|l|}{ Draining time, (days) } \\
\hline Mean \pm SD & $3.1 \pm 1.0$ & $2.5 \pm 0.9$ & 0.003 \\
\hline In-hospital complications & $2(5.2 \%)$ & $3(6.7 \%)$ & 1.000 \\
\hline Wound infection & $1(2.6 \%)$ & 0 & \\
\hline Intracranial infection & 0 & $1(2.2 \%)$ & \\
\hline Epilepsy & 0 & $1(2.2 \%)$ & \\
\hline Pneumonia & $1(2.6 \%)$ & $1(2.2 \%)$ & \\
\hline Mortality & $1(2.6 \%)$ & 0 & \\
\hline Recurrence within 3 month after surgery & $7(18.4 \%)$ & $5(11.1 \%)$ & 0.345 \\
\hline \multicolumn{4}{|l|}{ mRS score at 3-month follow-up } \\
\hline Mean \pm SD & $1.4 \pm 1.4$ & $1.3 \pm 1.1$ & 0.633 \\
\hline$\geq 4$ & $2(5.3)$ & $1(2.2 \%)$ & 0.591 \\
\hline \multicolumn{4}{|l|}{ GOS score at 3-month follow-up } \\
\hline Mean \pm SD & $4.6 \pm 0.8$ & $4.8 \pm 0.4$ & 0.197 \\
\hline$\leq 3$ & $2(5.3 \%)$ & 0 & \\
\hline
\end{tabular}
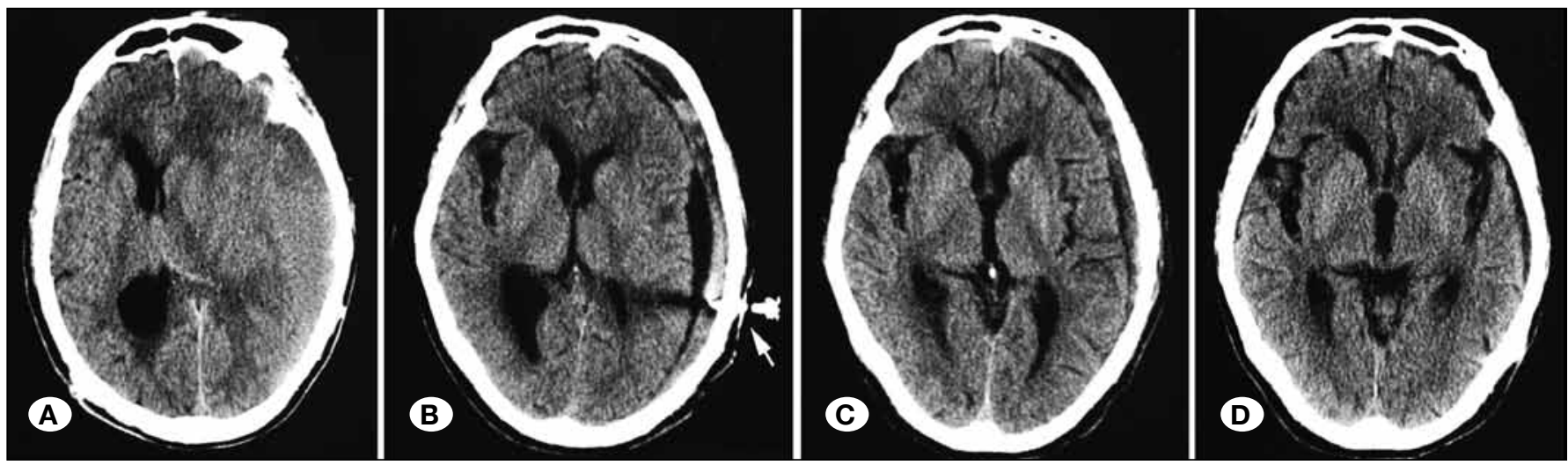

Figure 1A-D: Computed tomography scans of the patient in the illustrative case. The arrow denotes the location of the draining needle. 
the midline shift, a thin-layer of the residual hematoma, and reappearance of left insular gyruses (Figure 1C). He lived independently after discharge and got a mRS score of 0 at 3-month follow-up, when the head CT scan showed total absorption of the residual hematoma (Figure 1D).

\section{DISCUSSION}

Since the introduction of TDC technique three decades ago (15), several studies have compared this technique with BHC in the management of $\mathrm{CSDH}$, which resulted in controversial conclusions $(6,8,14,17)$. Smely et al. first conducted a prospective study including 33 patients in TDC and 33 patients in BHC treatment, and the result showed that TDC was superior to $\mathrm{BHC}$ due to a low recurrence rate $(18.1 \%$ vs. $33.3 \%)$, infection rate (0 vs. $18.1 \%)$, and mortality rate $(6.0 \%$ vs. $9.0 \%)(14)$. After that, a retrospective study from Williams et al. found a significantly higher recurrence rate in TDC than in BHC $(63.6 \%$ vs. $7.1 \%)$, hence in favor of TDC when treating the disease (17). This study showed that there was no significant difference in recurrence rate, in-hospital complications, and neurological outcome at 3-month followup between the patients who received TDC or BHC treatment.

In contrast to previous studies in which a draining tube was placed in the hematoma cavity when performing TDC $(5,7,12,14)$, here we used a modified technique to do the operation. After its tip penetrated the outer membrane of the hematoma, the hollow needle autonomously fixed on the cranium and served as a tunnel for drainage. This tool waived the time spent on insertion of a draining tube, further shortening the operating time. Free from tube insertion may also minimize the possibility of subdural air collection after surgery, which is recognized as a risk factor for CSDH recurrence $(10,11,13)$. The recurrence rate in the patients treated by this technique was $18.4 \%$ in this study, comparable to $18.1 \%$ in Smely's study and $18.2 \%$ in Horn's study in which a subdural draining tube was placed when performing TDC $(7,14)$. The comparison between this modified TDC technique and conventional technique need to be assessed by future studies.

This study showed that patients in TDC had significantly longer draining time than those in $\mathrm{BHC}$, which was consistent with the result from the study conducted by Muzii et al.(8). A longer draining time usually means a longer bedridden period for the patients, and this may raise the concerns of clinicians about postoperative complications. Elderly patients are more susceptible to complications of immobility than young patients, such as pneumonia and deep vein thrombosis. However, this study showed no significant difference in inhospital complications between the patients in TDC and those in BHC. Further studies with a larger sample size are warranted to verify these findings.

Another positive finding in this study was that patients in TDC had a smaller degree of midline shift reversal than those in $\mathrm{BHC}$. The procedure of $\mathrm{BHC}$ is more effective for midline shift reversal than TDC, especially for the patients with preoperative midline shift $\geq 5 \mathrm{~mm}$. Noteworthy, as for the eight patients with a preoperative GCS score $\leq 13$, one $(25.0 \%)$ of the four patients in TDC and three (75.0\%) of the four patients in BHC saw their GCS score improved to 14-15 after surgery. These findings may support the usage of $\mathrm{BHC}$ for the patients with severe midline shift $(\geq 5 \mathrm{~mm}$ ) or a lower GCS score before surgery.

Some limitations of this study should be acknowledged. This is a prospective study with a limited sample size, and therefore has sources of bias and variation. A prospective randomized controlled trial with a larger number of patients is required to verify the findings in this study. The patients enrolled in this study were also selected, which may limit extrapolation of the results in this study.

\section{CONCLUSION}

TDC and BHC have similar clinical outcomes in the treatment of patients with CSDH. A shorter operating time, but a smaller midline shift reversal and a longer draining time may be expected in patients after TDC than after BHC.

\section{ACKNOWLEDGEMENT}

This work was supported by the National Scientific Foundation of China (81201708) and the Foundation of Shanghai Municipal Public Health Bureau (20124299).

\section{- REFERENCES}

1. Asghar M, Adhiyaman V, Greenway MW, Bhowmick BK, Bates A: Chronic subdural haematoma in the elderly-a North Wales experience. J R Soc Med 95:290-292, 2002

2. Baechli H, Nordmann A, Bucher HC, Gratzl O: Demographics and prevalent risk factors of chronic subdural haematoma: Results of a large single-center cohort study. Neurosurg Rev 27:263-266, 2004

3. Cenic A, Bhandari M, Reddy K: Management of chronic subdural hematoma: A national survey and literature review. Can J Neurol Sci 32:501-506, 2005

4. Chon KH, Lee JM, Koh EJ, Choi HY: Independent predictors for recurrence of chronic subdural hematoma. Acta Neurochir 154:1541-1548, 2012

5. Escosa Bae M, Wessling H, Salca HC, de Las Heras Echeverria $P$ : Use of twist-drill craniostomy with drain in evacuation of chronic subdural hematomas: Independent predictors of recurrence. Acta Neurochir 153:1097-1103, 2011

6. Gokmen M, Sucu HK, Ergin A, Gokmen A, Bezircioglu H: Randomized comparative study of burr-hole craniostomy versus twist drill craniostomy; surgical management of unilateral hemispheric chronic subdural hematomas. Zentralbl Neurochir 69:129-133, 2008

7. Horn EM, Feiz-Erfan I, Bristol RE, Spetzler RF, Harrington TR: Bedside twist drill craniostomy for chronic subdural hematoma: A comparative study. Surg Neurol 65:150-153, 2006

8. Muzii VF, Bistazzoni S, Zalaffi A, Carangelo B, Mariottini A, Palma L: Chronic subdural hematoma: Comparison of two surgical techniques. Preliminary results of a prospective randomized study. J Neurosurg Sci 49:41-46, 2005 
9. Nakaguchi $\mathrm{H}$, Tanishima $\mathrm{T}$, Yoshimasu $\mathrm{N}$ : Factors in the natural history of chronic subdural hematomas that influence their postoperative recurrence. J Neurosurg 95:256-262, 2001

10. Nakaguchi $\mathrm{H}$, Tanishima T, Yoshimasu N: Relationship between drainage catheter location and postoperative recurrence of chronic subdural hematoma after burr-hole irrigation and closed-system drainage. J Neurosurg 93:791-795, 2000

11. Ohba S, Kinoshita Y, Nakagawa T, Murakami H: The risk factors for recurrence of chronic subdural hematoma. Neurosurg Rev 36:145-150, 2013

12. Ramnarayan R, Arulmurugan B, Wilson PM, Nayar R: Twist drill craniostomy with closed drainage for chronic subdural haematoma in the elderly: An effective method. Clin Neurol Neurosurg 110:774-778, 2008
13. Shiomi N, Sasajima H, Mineura K: Relationship of postoperative residual air and recurrence in chronic subdural hematoma. No Shinkei Geka 29:39-44, 2001

14. Smely C, Madlinger A, Scheremet R: Chronic subdural haematoma-a comparison of two different treatment modalities. Acta Neurochir 139:818-825, 1997

15. Tabaddor K, Shulmon K: Definitive treatment of chronic subdural hematoma by twist-drill craniostomy and closedsystem drainage. J Neurosurg 46:220-226, 1977

16. Weigel R, Schmiedek P, Krauss JK: Outcome of contemporary surgery for chronic subdural haematoma: Evidence based review. J Neurol Neurosurg Psychiatry 74:937-943, 2003

17. Williams GR, Baskaya MK, Menendez J, Polin R, Willis $B$, Nanda A: Burr-hole versus twist-drill drainage for the evacuation of chronic subdural haematoma: A comparison of clinical results. J Clin Neurosci 8:551-554, 2001 